\title{
Temperature measurement at the end of a cantilever using oxygen paramagnetism in solid air
}

\author{
Kent R. Thurber and Doran D. Smith \\ U.S. Army Research Laboratory, AMSRL-SE-EM, Adelphi, Maryland 20783 \\ Lee E. Harrell \\ Department of Physics, U.S. Military Academy, West Point, New York 10996
}

(Dated: July 2, 2018)

\begin{abstract}
We demonstrate temperature measurement of a sample attached to the end of a cantilever using cantilever magnetometry of solid air "contamination" of the sample surface. In experiments like our Magnetic Resonance Force Microscopy (MRFM), the sample is mounted at the end of a thin cantilever with small thermal conductance. Thus, the sample can be at a significantly different temperature than the bulk of the instrument. Using cantilever magnetometry of the oxygen paramagnetism in solid air provides the temperature of the sample, without any modifications to our MRFM (Magnetic Resonance Force Microscopy) apparatus.
\end{abstract}

In manorpanetic Resonance Force Microscopy

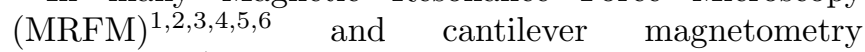
experiments, 7 the sample being measured is mounted at the end of a thin cantilever. The cantilever often has very poor thermal conductance because of its small cross section in comparison to its length. Even a small heat input into the sample can raise its temperature significantly from the temperature at the opposite end (base) of the cantilever. Obviously, the temperature of the sample can be critical to the results of an experiment because of many temperature dependent factors like spin polarization or time constants.23 Mounting a temperature sensor at the end of the cantilever would be difficult, requiring a sensor of $\leq 10 \mu \mathrm{m}$ dimensions integrated with the cantilever. An indirect way to measure the temperature of a cantilever is to measure the thermal (Brownian) motion of the cantilever. Using simple Brownian motion requires that the spring constant of the loaded cantilever is known and that the temperature is uniform along the cantilever and equal to the sample temperature. In addition, the Brownian motion method relies on the absence of non-thermal sources of cantilever motion for correct temperature measurement. Sources of sample heating (such as RF magnetic fields and optical power) can also be sources of non-thermal motion.

A simple alternative is to use cantilever magnetometry to measure the paramagnetism of oxygen in frozen air on the sample. This technique does not require any modification of the experimental probe used for MRFM or cantilever magnetometry, and provides a relative measure of the sample temperature up to the sublimation temperature of the solid air. In essence, the temperature is measured by using the temperature dependence of the magnetic susceptibility of the air "contamination" of the sample surface. The paramagnetic electron spins of the oxygen molecules in the solid air have a large, easily measureable temperature dependence of magnetic susceptibility. This technique could also be done just using the susceptibility of the sample itself if there is enough temperature dependence of the susceptibility.

The experimental setup is the same as used for our sample on cantilever MRFM experiments. The sample for MRFM (in this case GaAs) is mounted with thermally conductive silver epoxy on a gold-coated $\mathrm{SiN}_{x}$ cantilever with spring constant, $\mathrm{k} \sim 0.05 \mathrm{~N} / \mathrm{m}$. The $\mathrm{SiN}_{x}$ cantilever has very poor thermal conductance because of the low thermal conductivity of $\mathrm{SiN}_{x}$ and thin cross section (the cantilever is $0.6 \mu \mathrm{m}$ thick, $22 \mu \mathrm{m}$ wide, and $320 \mu \mathrm{m}$ long). 9 In order to increase the thermal conductivity of the cantilever, it was coated on each side with either 300 $\AA / 700 \AA$ or $300 \AA / 1700 \AA$ of $\mathrm{Ti} / \mathrm{Au}$. Coating on both sides is required to reduce bending of the cantilever from the differential contraction of the gold and $\mathrm{SiN}_{x}$ on cooling. The position of the cantilever is measured with an optical interferometer. 3 Below the sample is the source of the magnetic field gradient, a $250 \mu \mathrm{m}$ diameter iron cylinder. The magnetic field gradient $\left(\delta B_{z} / \delta z=6000\right.$ to $9000 \mathrm{~T} / \mathrm{m})$ applies a static force,

$$
F_{z}=\mu_{z} \frac{\delta B_{z}}{\delta z}
$$

to the net magnetic moment of the oxygen electrons, $\mu$, which we measure by the deflection of the cantilever. Raw data from the interferometer is shown in figure 1. As the magnetic field is increased, the oxygen electrons become more polarized and the force between the oxygen and the magnetic field gradient becomes larger. The cantilever deflects towards the iron magnet. Raising the temperature decreases the susceptibility and thus the amount of deflection (figure 1(c)).

This technique of temperature measurement could be done with the sample itself, if the sample has sufficient temperature dependence of its magnetic susceptibility for easy measurement. However, "contamination" of the sample and cantilever surface with solid air can be used with an arbitrary sample. In addition, air is a resource available in almost every lab. Coating the sample with a small amount of frozen air can easily be done by not fully pumping out the probe before cool down. Because of the 


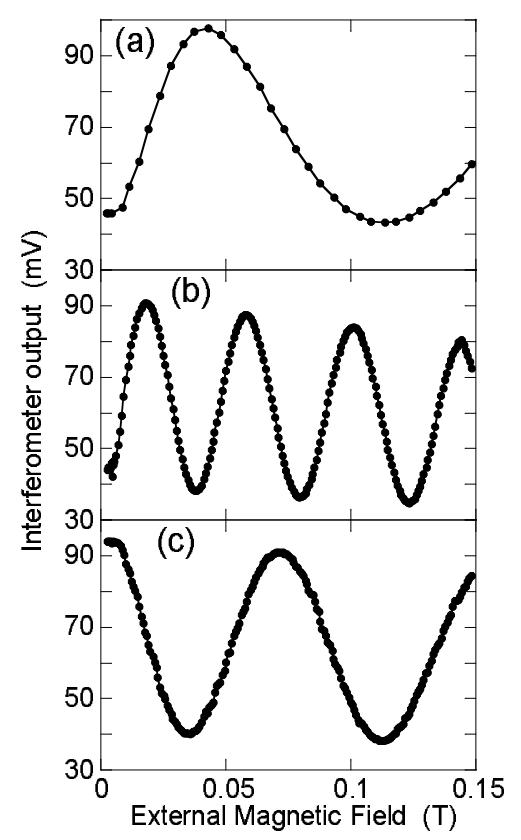

FIG. 1: Optical interferometer measurement of the cantilever position as a function of external magnetic field for (a) 4.2 $\mathrm{K}$ without air frozen on sample, (b) $4.2 \mathrm{~K}$ with frozen air on sample, and (c) $8.4 \mathrm{~K}$ with frozen air on sample. Each interferometer oscillation represents $\lambda / 2=390 \mathrm{~nm}$ of cantilever deflection.

sensitivity of the cantilever, very little air is required. Just $10^{11}$ oxygen molecules ( $\sim$ monolayer) will result in several interferometer fringes of deflection (hundreds of $\mathrm{nm}$ ) during a magnetic field sweep to $9 \mathrm{~T}$. Deliberately allowing air into the vacuum system with a non-regulating valve typically resulted in too much air contamination and the magnetic force would stick the cantilever to the iron magnet.

This provides a cautionary warning for cantilever magnetometry or MRFM experiments. Oxygen contamination must be avoided for sensitive magnetometry or MRFM experiments because of the large magnetic susceptibility of oxygen. For this reason, the temperature measurements were done first and then the air contamination removed before the MRFM experiments. The air contamination could be removed by heating the probe head to about $20 \mathrm{~K}$ while pumping. The best technique for avoiding air contamination was to use the heater to keep the probe head warm, while the rest of the probe was cooled down.

We should also note that solid air is a better choice for large and simple temperature dependence of the magnetic susceptibility than pure oxygen. Pure solid oxygen has antiferromagnetic interactions between the molecules and undergoes two phase transitions in the solid state at low temperatures (43.8 and $23.9 \mathrm{~K}$ ) 10.11 By contrast, the oxygen electrons in solid air have been studied recently with EPR at $5 \mathrm{~K}$ and shown to behave as $S=1$

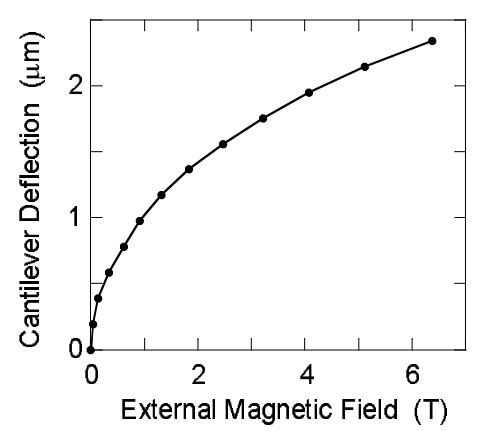

FIG. 2: Cantilever deflection as a function of external magnetic field for air "contaminated" sample at $4.2 \mathrm{~K}$.

paramagnets 10 following the Hamiltonian

$$
H=g \mu_{B} B S_{z}+D\left(S_{z}^{2}-\frac{S(S+1)}{3}\right)
$$

where $g=2.0$ and the zero field splitting $D=5.1 \mathrm{~K}$. The Hamiltonian is written for the case where the axis of the oxygen molecule is along the magnetic field. In the frozen air, the oxygen molecules have a distribution of their molecular axes with respect to the magnetic field. 10 Apparently, the oxygen molecules are dispersed enough among the nitrogen molecules in the solid air to prevent antiferromagnetic ordering. Similarly, dilute adsorbed oxygen results in roughly Curie-Weiss law behavior of the susceptibility, $1 /(T-\theta)$, with $\theta$ small and negative.11 Figure 2 shows the deflection of the cantilever up to high field. The saturation of the susceptibility at high field is consistent with spin 1 and $g \approx 2$ electronic moments. To provide a calibration for this temperature measurement method, we heated the probe head, which results in a nearly uniform temperature of the probe head, cantilever, and sample. The probe head itself has its temperature measured with a resistive sensor. 12 As shown in figure 3 , the temperature dependence of the magnetic susceptibility does indeed roughly follow a Curie-Weiss law with the fitted $\theta=-2 \mathrm{~K}$.

Then, we applied this technique to measure the sample temperature under conditions when it is different from the temperature of the rest of the probe head. The magnetic susceptibility of the sample with air "contamination" is measured by the deflection of the cantilever by a change in magnetic field. The temperature is derived by comparison with the calibration done by heating the entire probe head. The calibration step could be avoided by assuming the Curie-Weiss law temperature dependence of the oxygen susceptibility and the dominance of the oxygen susceptibility over any other magnetic susceptibility of the sample or cantilever. In these MRFM experiments, the GaAs sample can be heated from two major sources. First, we are applying RF magnetic field which can heat the sample and gold-coated cantilever directly by eddy current heating and indirectly through gas conduction. Second, and more significantly, we are 


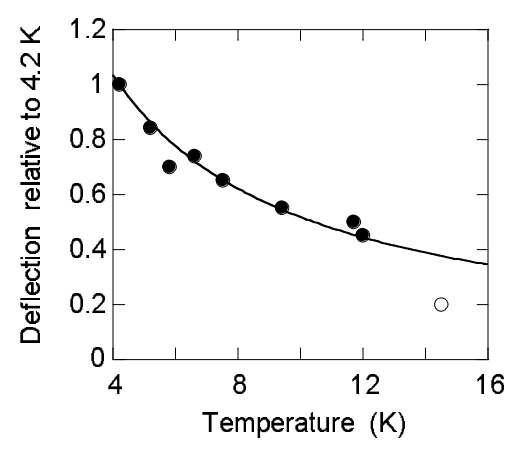

FIG. 3: Oxygen magnetometry as a function of temperature. The oxygen magnetometry, $\bullet$, is plotted as the deflection of the cantilever over a magnetic field sweep $(0.15,0.8$, or $2 \mathrm{~T})$ relative to the deflection over the field sweep at $4.2 \mathrm{~K}$. At the highest temperature ( $\circ 14.5 \mathrm{~K}$ ), oxygen desorbs when under low pressure and the magnetometry is no longer reversible with temperature. The line is a Curie-Weiss law fit to the data below $14.5 \mathrm{~K}(\theta=-2 \mathrm{~K})$

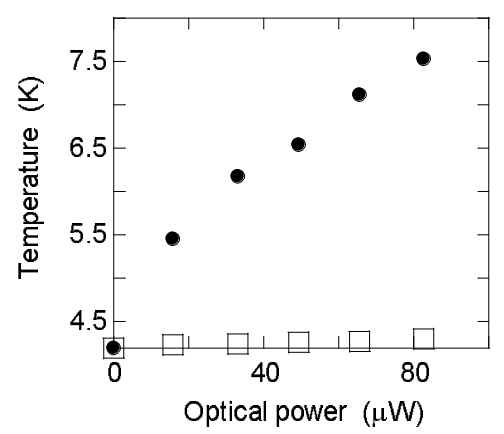

FIG. 4: Comparison of sample temperature measured by oxygen magnetometry $(\bullet)$ and the temperature of the bulk of the probe $(\square)$ while shining $823 \mathrm{~nm}$ light on the sample. Cantilever is coated on both sides with $300 \AA$ Ti and $700 \AA \mathrm{Au}$. shining light on the sample for optical pumping.13 Another smaller heat source is the optical power used in the interferometer measurement. We measured the susceptibility and found, for instance, that roughly 4 Gauss of $\mathrm{RF}(51.5 \mathrm{MHz})$ magnetic field raised the sample temperature about $1 \mathrm{~K}$. Even more dramatic is the effect of optical pumping (Fig. 4). The $\sim 80 \mu \mathrm{W}$ of optical power on the GaAs sample at the end of the cantilever can heat it by $3 \mathrm{~K}$, while the main bulk of the probe has warmed by less than $0.1 \mathrm{~K}$. This local heating is consistent with the estimated thermal conductance of the cantilever, $\approx 25$ $\mu \mathrm{W} / \mathrm{K}$, which is dominated by the $700 \AA$ gold coating.

We have presented a technique for measuring the temperature of a sample at the end of a cantilever. In general, oxygen contamination must be avoided for cantilever magnetometry and MRFM experiments. But here, by measuring the susceptibility of the oxygen in solid air contamination, we can determine the temperature without any contact to the sample. This technique can be extended by using the susceptibility of some other "contaminant" or just the sample itself. In addition, these measurements are done without any modification of the apparatus which we use primarily for MRFM measurement of the nuclear polarization of the sample.

This work was supported by the DARPA Defense Science Office Spins in Semiconductors program. The cantilevers were coated by Monica Taysing-Lara. The authors would like to thank John A. Marohn, John Sidles, and Dan Rugar for many helpful discussions.
${ }^{1}$ K. R. Thurber, L. E. Harrell, R. Fainchtein, and D. D. Smith, Appl. Phys. Lett. 80, 1794 (2002).

${ }^{2}$ K. Wago, O. Züger, R. Kendrick, C. S. Yannoni, and D. Rugar, J. Vac. Sci. Technol. B 14, 1197 (1996).

3 D. D. Smith, J. A. Marohn, and L. E. Harrell, Rev. Sci. Instrum. 72, 2080 (2001).

4 O. Klein, V. V. Naletov, and H. Alloul, Eur. Phys. J. B 17, 57 (2000).

5 K. J. Bruland, J. Krzystek, J. L. Garbini, J. A. Sidles, Rev. Sci. Instrum. 66, 2853 (1995).

${ }^{6}$ R. Verhagen, A. Wittlin, C. W. Hilbers, H. van Kempen, and A. P. M. Kentgens, J. Am. Chem. Soc. 124, 1588 (2002).
7 J. G. E. Harris, D. D. Awschalom, F. Matsukura, H. Ohno, K. D. Maranowski, and A. C. Gossard, Appl. Phys. Lett. 75, 1140 (1999).

${ }^{8}$ H. J. Mamin and D. Rugar, Appl. Phys. Lett. 79, 3358 (2001).

9 MLCT-NOHW Microcantilevers, Park Scientific Instruments, Sunnyvale, CA.

${ }^{10}$ L. A. Pardi, J. Krzystek, J. Telser, and L.-C. Brunel, J. Magn. Reson. 146, 375 (2000).

11 Stephen Gregory, Phys. Rev. Lett. 39, 1035 (1977).

12 Germanium Cryo Resistor, TRI Research.

13 G. Lampel, Phys. Rev. Lett. 20, 491 (1968). 